\title{
Investigación cualitativa y análisis de contenido temático. Orientación intelectual de revista Universum
}

\author{
Claudio Díaz Herrera ${ }^{1}$
}

Recibido: 16 de octubre 2017 / Aceptado: 30 de enero de 2018

Resumen. Respondiendo a una problemática técnica y metódica que permita identificar alguna orientación temática o intelectual en un medio de difusión académica, el artículo propone un sustento teórico-metodológico, que aporte hacia la elaboración procedimental de un análisis de contenido temático y cualitativo para estos fines investigativos. La propuesta puede ser aplicada en estudios donde sea factible constituir longitudinalmente una trayectoria de información, expresada en formatos de producción científica, como por ejemplo revistas periódicas, actas de congreso, catálogos de bibliotecas, etc. Lo anterior, considerando la existencia de riqueza multidisciplinaria o cierta diversidad temática contenida en estos medios de difusión. En términos empíricos la propuesta teórica-metodológica se aplicó en revista Universum, que al cumplir 30 años de edición, su génesis se caracteriza por una composición miscelánea, determinando un tránsito por diversas temáticas de estudio hasta indexarse científicamente, hecho que conllevó su adscripción al área de humanidades y ciencias sociales. La propuesta se transforma en un procedimiento válido para en este caso, constituir la orientación temática de una revista. A la luz de los resultados, se concluye que Universum se orienta predominantemente hacia cuatro etiquetas centrales, en primer lugar una dimensión de humanismo y letras; en segundo lugar, una dimensión de teoría social, política y cultural; continuando con una dimensión en desarrollo económico y administración; para terminar con estudio de las ideas. Finalmente, los procedimientos cualitativos aplicados, aportan técnicamente a la construcción de etiquetas/dimensiones, que una vez sistematizadas, categorizadas y re-categorizadas, dan cuenta de una orientación temática en este medio de difusión.

Palabras clave: Investigación cualitativa; análisis de contenido; categorización temática; humanidades; ciencias sociales; revista Universum.

\section{[en] Qualitative research and thematic content analysis. Intellectual orientation of Universum journal}

\begin{abstract}
Responding to a technical and methodical problem that allows to identify some thematic or intellectual orientation in a means of academic dissemination, the article proposes a theoreticalmethodological support, which contributes towards the procedural elaboration of an analysis of thematic and qualitative content for these investigative purposes. The proposal can be applied in studies where it is feasible to constitute an information trajectory longitudinally, expressed in scientific production format, as periodical journals, conference proceedings, library catalogs, etc. The

1 Universidad de Talca. Instituto de Estudios Humanísticos “Juan Ignacio Molina”. Doctorado en Ciencias Humanas.

E-mail: cldiaz@utalca.cl
\end{abstract}


foregoing, to the extent that there is multidisciplinary wealth or a certain thematic diversity contained in these media. In empirical terms, the theoretical-methodological proposal was applied in the Universum journal, that after 30 years of editing, its genesis is characterized by a miscellaneous composition, determining a transit through diverse thematic of study until being indexed scientifically, fact that led to his affiliation to the humanities and social sciences area. The proposal becomes a valid procedure to constitute the thematic orientation of a journal. In light of the results, it is concluded that Universum is predominantly oriented towards four central labels, first a dimension of humanism and letters; secondly, a dimension of social, political and cultural theory; continuing with a dimension in economic development and administration; to end with history of ideas. Finally, the qualitative procedures applied, contribute technically to the construction of labels / dimensions, which once systematized, categorized and re-categorized, give an account of a thematic orientation in this diffusion medium.

Keywords: Qualitative research; content analysis; thematic categorization; humanities; social sciences; Universum journal.

Sumario. 1. Introducción; 2. Metodología; 3. Resultados; 4. Discusión; 5. Conclusiones;6. Agradecimientos; 7. Referencias Bibliográficas.

Cómo citar: Díaz Herrera, C. (2018) Investigación cualitativa y análisis de contenido temático. Orientación intelectual de revista Universum, en Revista General de Información y Documentación 28 (1), 119-142.

\section{Introducción}

El artículo constituye un trabajo cuya preocupación es la constante validación de los procedimientos metodológicos, sobre todo desde una perspectiva cualitativa. Esto en el entendido que se debe constantemente fortalecer y aportar hacia la construcción del conocimiento, validando los procedimientos que permiten una elaboración sólida y fundamentada del quehacer investigativo.

En este sentido, Devés (2016) da cuenta de la necesidad de realizar una evaluación a la producción intelectual en ciencias sociales, con estrategias dentro de las cuales considera cantidad de publicaciones, evaluar la trayectoria de este trabajo, es decir, la forma en como una trayectoria de científicos sociales han generado una densidad intelectual a través de un quehacer conceptualizado del área de estudio y que permita generar nuevas problemáticas e ideas que logren trascender y proyectarse en el tiempo.

En este sentido y con el objeto de generar una propuesta teórica y metodológica que permita ayudar a la identificación de una orientación intelectual o temática de un medio de difusión académico, desde una perspectiva cualitativa de análisis de contenido temático, el presente texto se elabora en primera instancia a partir de capítulos a modo de revisión bibliográfica, los que contextualizan un tránsito desde la epistemología de investigación, hasta el enfoque de investigación cualitativo sin dejar de discutir le emergencia de los métodos mixtos, para finalizar teóricamente con el análisis de contenido temático. Este recorrido, pretende ubicar al lector en el devenir teórico de la investigación social, que permita fundamentar en términos procedimentales la aplicación de un análisis de contenido temático y cualitativo, tal como se expresa en el desarrollo empírico del trabajo. 


\subsection{Distinciones paradigmáticas y sus respectivos enfoques metodológicos}

La discusión entre enfoques cuantitativos o cualitativos de investigación social son de larga data. Ya a comienzos de la sociología en el siglo XIX y comienzos del $\mathrm{XX}$, la importancia de las teorías funcionalistas al alero de los métodos de investigación social cuantitativos, determinan la preponderancia y orientación de la investigación (Ritzer, 2001). De esta forma, el desarrollo de las ciencias sociales ha ido de la mano con los contextos sociales, políticos y económicos de las sociedades, circundando teorías críticas, funcionalistas y sus respectivos métodos de investigación (De Sierra, Garretón, Murmis, \& Trindade, 2007).

Según lo anterior, parece pertinente comenzar recogiendo la distinción que plantea Guba y Lincoln (2002) en relación con las preguntas esenciales de acercamiento al conocimiento. Estas refieren a una dimensión ontológica, epistemológica y metodológica, que nos permitirían dar luces de distinciones epistémicas que sustentan el cómo se construye el conocimiento.

Complementando la postura anterior de los autores mencionados, Cisterna (2005) citando a Habermas (1986) plantea que, en relación a la configuración de la ciencia bajo estructuras que no son ideológicas ni políticamente neutrales, existe una relación entre el conocimiento y el interés. Esto determinaría para el autor, la distinción entre un interés técnico, otro práctico y finalmente uno emancipatorio, los que a su vez se expresan en sus correspondientes paradigmas y enfoques de investigación. Para el interés técnico se asocia el neopositivismo con enfoque empirista y cuantitativa; a partir del interés práctico deriva una ciencia hermenéutica por tanto cualitativa; y del interés emancipatorio, deviene una ciencia socio - crítica, la cual utiliza procedimientos cualitativos, pero también complementados con resultados cuantitativos.

Con estas distinciones, emerge una complejidad que reviste la metodología en ciencias sociales, que da cuenta de una diversidad de elementos que debemos considerar a la hora de realizar investigación. Entre ellos, resulta pertinente reconocer en las ciencias sociales un área del conocimiento multiparadigmática como lo plantearía Giddens (1999), Habermas (1989) o Ritzer (2002) entre otros. Los autores convergen al plantear que el saber se vuelve un conocimiento a partir de múltiples formas de posicionarse ante los fenómenos de estudio, cuál de ellos más o menos interpretativos, lo que puede llegar a distar de la neutralidad o cientificismo newtoniano. Lo anterior, independiente de la interesante discusión de esta clásica aseveración supuestamente multiparadigmática realizada por Noguera (2010).

En definitiva, consideramos aquí ciertas posiciones paradigmáticas que nos permiten de alguna forma acercarnos al conocimiento, y que tendrían desde una posición asumida por el investigador -así como el tipo de problema que se desea investigar-, una respectiva forma (método) de enfrentar y abordar esta vacío de conocimiento a dilucidar. 


\subsection{Paradigma de investigación}

A partir de lo anterior, debemos destacar que existe una problemática que enfrentan históricamente las disciplinas científicas hoy en día, sobre todo desde la tribuna de las ciencias sociales y humanidades, las que deben por definición velar por posicionarse de una u otra forma respecto a un fenómeno a estudiar u objeto de estudio.

Así tenemos por un lado el positivismo científico y filosófico, el cual dominó Europa durante la segunda mitad del siglo XIX. Pero finalizando el mismo siglo referido, emergen diversas corrientes del pensamiento que se erigen como una respuesta a la dominación positivista en la forma cómo se configura el conocimiento. Cambia por tanto la concepción positivista reconociendo sus propios límites (Tejedor, 1993). El mismo autor sostiene en consecuencia que, Mach y Hertz formulan principios anti positivistas como por ejemplo "1) los conceptos científicos no son sino «signos» (y no «copias») de la realidad; 2) las leyes científicas no son sino «instrumentos» de previsión de los fenómenos” (pág. 372). De esta forma toma relevancia una nueva corriente filosófica, la cual propone comenzar a destacar concepciones referentes al espíritu, la libertad, los valores y la historia las que merecen ser estudiadas en profundidad debido a su complejidad conceptual y epistémica de abordaje.

Para Tylor \& Bogdan (1987), en ciencias sociales existió un predominio positivista con sus respectivas leyes de la naturaleza, así como modelos objetivos de explicación de los fenómenos sociales. Por su parte, Comte y Durkheim fueron los responsables de iniciar en el mundo de la sociología dicha forma de explicar los fenómenos. Luego en una segunda etapa, surge con fuerza una postura más fenomenológica y comprensiva, con el objeto de entender dichos fenómenos con autores como Husserl y Schutz, según refiere Ritzer (2001), (2002).

A partir de ahí, dicha postura puede ayudarnos a distinguir un paradigma "constructivista" e "interpretativista", que a juicio de Valles (2000) asumiría la existencia de realidades múltiples que se diferenciarían del positivismo. De esta forma, y alejándonos del positivismo según Dilthey en Stake (1999), la ciencia necesitaba más allá de explicaciones de la naturaleza, una comprensión del ser humano de sí mismo, en honor a lo experencial y los sentimientos que circundan en él.

Por tanto, los estudios cualitativos poseen una raíz weberiana, la cual analiza de una forma comprensiva al actor social. Esto porque el sujeto es capaz de interpretar mediante esta compresión o Verstehen la acción social en su propio contexto (Weber, 2005). Ahora bien, la decisión por un enfoque de investigación, no es una elección deliberada, irracional o de sentido común para el investigador, sino que surge a partir de procedimientos metodológicos sustentados en una episteme que definirá y orientará la elección metodológica (Valles, 2000).

Entendiendo estas distinciones epistémicas que derivan hacia enfoques metodológicos, para algunos investigadores, los métodos de investigación social resultan antagónicos según sus respectivos enfoques de investigación, discusión a la cual es deber referirse según el incremento de estudios multi método en la actualidad. 


\subsection{La superación de la disputa de enfoques metodológicos: Estudios mixtos}

En relación a lo anterior, la distinción entre los paradigmas de investigación que asumen enfoques metodológicos respectivos (Guba \& Lincoln, 2002), se ha visto superada en términos supuesta relevancia o supremacía a la hora de investigar de uno, por sobre otro enfoque metodológico.

Si bien en estudios incipientes, cuando investigadores se están iniciando en el mundo de la investigación social, resulta pertinente reconocer la historicidad y trayectoria epistémica de la generación del conocimiento que llega a constituir diferentes enfoques de investigación, y con el objeto de evitar incertidumbre derivada de esta discusión de posicionamiento epistémico investigativo para construir conocimiento (Díaz, 2014), hoy por hoy, se plantea como superada esta disputa metodológica cualitativa y cuantitativa. Métodos de investigación que alguna vez fueran considerados antagónicos para la construcción del conocimiento, hoy se expresan como una discusión poco fructífera y relevante (Hernández, Fernández, \& Baptista, 2010).

En este sentido, toma realce la combinación de enfoques metodológicos los cuales se pueden identificar bajo diferentes nombres, como bien sistematiza Hernández, Fernández, \& Baptista (2010, pág. 546): “investigación integrativa (Johnson y Onwuegbuzie, 2004), investigación multimétodos (Hunter y Brewer, 2003; Morse, 2003), métodos múltiples (M. L. Smith, 2006; citado por Johnson, Onwuegbuzie y Turner, 2006), estudios de triangulación (Sandelowski, 2003), e investigación mixta (Tashakkori y Teddlie, 2009; Plano y Creswell, 2008; Bergman, 2008; y Hernández Sampieri y Mendoza, 2008)”.

Para los autores, la idea de este enfoque es aportar e integrar desde dos miradas o enfoques metodológicos diferentes a un estudio en particular. Esto con el objeto de enriquecer y complementar el conocimiento que se puede generar, abordando diferentes posturas y realzando algunos ámbitos que los enfoques por sí solo, resultarían deficientes para desentrañar la realidad social y cultural.

\subsection{Enfoque cualitativo de investigación}

Valles (2000) refiriéndose a Hamilton, plantea que la historia de los métodos cualitativos no son reductibles a intelectuales únicos. Por otro lado, estos orígenes se pueden encontrar en la ruptura kantiana de la "Crítica de la razón pura", al dominio cartesiano del objetivismo, tomando relevancia en Kant la interpretación y comprensión de los fenómenos por medio de subjetivismo, idealismo o relativismo.

Serían de corte etnográficas las corrientes que a partir del siglo XV, disciplinas como sociología y antropología, dan punto de partida a los métodos cualitativos, distinguiéndose por la disposición a desentrañar la existencia de un "otro" que comienza a descubrirse según la mirada colonialista (Valles, 2000). Así mismo, el autor refiriéndose a Vidich y Lyman (1994), plantea otro origen de lo cualitativo con el interés hacia el surgimiento de guettos norteamericanos, producto de la influencia calvinista y protestante. Por otro lado, para comienzos del siglo XX, influencia el contexto de la gran depresión económica, que desde la escuela de Chicago emerge un 
interés en estudios etnográficos, influenciados por la crisis económica y su efecto en el espacio urbano. Según Tylor \& Bogdan (1987) refiriéndose a Wax, los orígenes de las técnicas cualitativas están en el período clásico de Heródoto y con las incursiones económicas de Marco Polo, por tanto hay interés en la conversación con los sujetos (el otro), observación de manifestaciones culturales no eurocéntricas y una sistematización de información, gracias a las vivencias de estos incontables recorridos que fueron plasmados en bitácoras de viaje.

De esta forma, podemos dar cuenta que los estudios cualitativos no velan necesariamente por una medición numérica, sino que por descubrir expresiones culturales y sociales a través de un proceso interpretativo entre observador y observado (Hernández, Fernández, \& Baptista, 2010). Por otro lado, los métodos cualitativos:

"estudian la realidad en su contexto natural, tal y como sucede, intentando sacar sentido de o interpretar los fenómenos de acuerdo a los significados que tienen para las personas implicadas. La investigación cualitativa implica la utilización y recogida de una gran variedad de materiales, entrevistas, experiencia personal, etc., que describen la rutina, las situaciones problemáticas y los significados en la vida de las personas” (Flores, García, \& Rodríguez, 1996, pág. 10).

El estudio cualitativo apela a una observación próxima y detallada del sujeto en su propio contexto, para lograr aproximarse lo más posible a la significación de los fenómenos. Debiese cumplir según Ruiz (2012), con ciertas características propias desde la reconstrucción de significados, intentando interpretar y captar significados particulares y relevantes a los hechos, de manera metafórica y conceptual a partir del relato de los sujetos.

Según Bonilla y Rodríguez en Bernal (2006), los métodos cualitativos también son denominados como "no tradicionales", y estarían orientados a profundizar sin pretender generalizar dichos resultados, sino que a describir fenómenos por medio de los propios rasgos particulares, según sean percibidos en su contexto, por tanto, no pretenden medir, sino que cualificar estos hallazgos.

Colby en Hernández, Fernández, \& Baptista (2010), plantea que el común denominador de los estudios cualitativos son los patrones culturales, donde son los seres humanos quienes ven el mundo diferente de acuerdo a su cultura, a través de ciertas particularidades que son interpretados de forma diferente. Este argumento nos invita a relacionar los planteamientos mencionados de Wax (en Valles, 2000), en razón al valor de culturas desconocidas y la forma en cómo pueden connotar la realidad donde habitan. Por tanto, los estudios cualitativos no posee una génesis determinista, ya que, están enriquecidas por historias y aportes variados en su evolución (Stake, 1999).

Por su lado, para Ray Rist citado en Tylor (1987), lo central en las metodologías cualitativas se resumen en que son principalmente de tipo: descriptivas, inductivas, holísticas, sensibles, no intrusivas; comprensivas, analiza fenómenos como si fuesen la primera vez, acepta lo valioso de diferentes perspectivas, es humanista, se 
ajustan a validez empírica, todo es campo social, el sujeto es digno de estudio y termina por ser un verdadero arte.

\subsection{Análisis de contenido cualitativo}

Tenemos una aproximación hacia una necesidad de abordar cualitativamente técnicas utilizadas en el análisis de textos y documentos. Nos referimos así al análisis de contenido cualitativo, el cual puede apuntar al desarrollo complementario y paralelo en otros tipos de análisis de contenido, de impronta cuantitativa, y que en definitiva poseen otros objetivos. Por tanto y en palabras de Ruíz (2012), el análisis de contenido abarca una gama amplísima de conceptos, técnicas y contenidos que es preciso delimitar.

A juicio de Arbeláez \& Onrubia (2014), existirían diversas aproximaciones teóricas y metodológicas en el análisis de contenido que permiten de manera válida, interpretar textos y documentos de forma explícita o implícita. Los autores refiriéndose a Piñuel (2002), plantean que el análisis de contenido lo que revela es en definitiva el sentido que emerge del texto. Andrews (2002) en este mismo texto, lo expone como información expresa y latente, siendo la primera de ellas lo que plantea un determinado autor de manera explícita; y la información latente, lo que dice este autor sin pretenderlo de manera expresa, cobrando sentido sus planteamientos siempre desde un contexto determinado.

El "contenido de un texto no es algo que estaría localizado dentro del texto en cuanto tal, sino fuera de él, en un plano distinto en relación con el cual ese texto define y revela su sentido" (Delgado \& Gutiérrez, 1998, pág. 179). Según Araya (2002) y siguiendo la línea teórica de Bardin, se enfatiza el análisis de contenido en las dimensiones ocultas, el sentido latente y la inferencia a partir del relato emanado por los sujetos. En relación a lo anterior, el análisis de contenido cualitativo no tiene como fin solo la búsqueda de ciertos contenidos dentro de un corpus, sino de encontrar el sentido que estos contenidos, poseen dentro del contexto.

Para Marradi, Archenti, \& Piovani (2007, pág. 290) el análisis de contenido es "una técnica de interpretación de textos [...] que se basan en procedimientos de descomposición y clasificación de éstos (Losito 1993) [...] los textos de interés pueden ser diversos: trascripciones de entrevistas, protocolos de observación, notas de campos, fotografías, publicidades televisivas, artículos de diarios y revistas, discursos políticos, etcétera".

Bardin, siendo uno de los referentes en análisis de contenido, lo define como "un conjunto de técnicas de análisis de comunicación tendente a obtener indicadores (cuantitativos o no) por procedimientos sistemáticos y objetivos de descripción del contenido de los mensajes, permitiendo la inferencia de conocimientos relativos a las condiciones de producción / recepción (variables inferidas) de estos mensajes” (2002, pág. 32).

Según las descripciones conceptuales que hemos abordado como análisis de contenido, emergen elementos centrales y prioritarios en este tipo de análisis, nos referimos así a la interpretación y la emergencia de la inferencia. En esta dirección, Ruiz (2012) plantea que al ser la inferencia uno de los elementos centrales del 
análisis de contenido, la información emanada por los discursos o textos, posee sentidos los que pueden ser manifiestos o no, por lo que la comunicación simbólica expresa contenidos inferenciales, con patrones de significados los cuales pueden ser interpretados como indicadores o contextos.

Sin embargo y a pesar de lo cuestionable que para algunos más orientados al positivismo puede llegar a ser el valor de la interpretación e inferencia, el autor explica que el análisis de contenido vela por una lectura que debe realizarse por medio del método científico, es decir, de manera sistemática, objetiva, replicable, válida, siendo en consecuencia al igual que otros métodos semejantes en su problemática y metodología (Ruíz, 2012).

Para Arbeláez \& Onrubia (2014, pág. 19), el objeto del análisis de contenido cualitativo es "verificar la presencia de temas, palabras o de conceptos en un contenido y su sentido dentro de un texto en un contexto". Estos mismos autores refiriéndose a Hostil (1969) y Stone, Dunphy, Smith y Oglive (1966) consideran el análisis de contenido cualitativo como "una técnica de investigación para formular inferencias identificando de manera sistemática y objetiva ciertas características específicas dentro de un texto" (pág. 19). Con esto volvemos a connotar como la inferencia, pasa a jugar un papel preponderante lo que se relaciona a elementos subjetivos e interpretativos del análisis, inferencia que acuña Krippendorf (2002) y Bardin (2002) al alero de la importancia que reviste la existencia de este análisis dentro de un contexto, tal como ya hemos referido en palabras de Andréu (2000).

Respecto a los dos enfoques metodológicos en el análisis de contenido, el análisis de contenido cuantitativo apunta hacia una reducción de categorías analíticas, las que pueden determinarse en distribuciones frecuenciales y correlaciones entre otras, a diferencia de los análisis de contenido cualitativos, los cuales interpretan el contenido apoyándose de categorías analíticas para describir sus particularidades según Landry 1993 citado por Arbeláez \& Onrubia (2014). Los autores enfatizan si, que estos enfoques pueden llegar a ser complementarios más que antagónicos.

Para Andréu (2000, pág. 22), El análisis de contenido cualitativo “consiste en un conjunto de técnicas sistemáticas interpretativas del sentido oculto de los textos". La idea es desarrollar la perspectiva interpretativa de los textos, profundizando más allá de del contenido manifiesto, también al contexto y contenido latente desde donde se expresa el mensaje. El autor refiere que las ventajas que reviste este tipo de análisis cualitativo, podemos destacar el análisis en un modelo comunicativo; la consecución de reglas y procedimientos; creación de categorías centrales de análisis; y generación de constantes criterios de validez.

Según Andréu (2000), existirían dos orientaciones de análisis de contenido cualitativo, la primera de ellas mediante el desarrollo de categorías inductivas y la segunda de aplicación de categorías deductivas. Esto ante la poca claridad de la procedencia y desarrollo de estas categorías. El primero de ellos se centra en procedimientos reductivos de las categorías, con el objeto de crear criterios de definición, las cuales se van deduciendo sistemáticamente hasta lograr una categoría central. En el segundo tipo deductivo, estas se formulan desde la teoría, 
generando un libro codificado en el cual se van asociando estas categorías deductivas.

Debemos distinguir etapas en las técnicas del análisis de contenido según Arbeláez \& Onrubia (2014):

1. Fase teórica: Pre análisis. En el cual se organiza la información a través de una revisión superficial de los documentos, lo que permite la emergencia de las primeras aproximaciones hipotéticas del trabajo.

2. Fase Descriptiva - analítica. Donde se describen y analizan los artículos.

3. Fase interpretativa. Paso en el que se interpreta el análisis de contenido según las categorías emergentes de la producción académica publicada en la revista.

1.6 Análisis de contenido por categorización.

Según Bardín (2002, pág. 90), la categorización:

"es una operación de clasificación de elementos constitutivos de un conjunto por diferenciación, tras la agrupación por género (analogía), a partir de criterios previamente definidos. Las categorías son secciones o clases que reúnen un grupo de elementos (unidades de registro en el caso del análisis de contenido) bajo un título genérico, reunión efectuada en razón de dos caracteres comunes de estos elementos".

En la categorización, según Delgado \& Gutiérrez (1998, pág. 193) se debe:

"efectuar una clasificación de las unidades de registro (previamente codificadas e interpretadas en sus correspondientes unidades de contexto) según las similitudes y diferencias que sea posible apreciar de acuerdo a ciertos criterios, las cuales pueden ser sintácticas (nombres, verbos, adjetivos, etc.), semánticas (temas, áreas conceptuales, etc.), o pragmáticas (actitudes, formas de uso del lenguaje, etc.)".

El proceso de análisis, a partir de las unidades de registro, tiene sentido en la medida que "cada unidad de registro es un tipo de segmento textual claramente discernible y cuya ejemplificación en el corpus puede ser detectada" (Delgado \& Gutiérrez, 1998, pág. 192).

Según Delgado \& Gutiérrez (1998, pág. 193): “Es necesario no sólo detectar la unidad de registro, sino también localizarla. Con vistas a esta localización, las unidades de registro suelen referirse a lo que se llama unidades de contexto, la cual es un marco interpretativo de la relevancia de las unidades de registro detectadas". De esta forma, según el mismo autor: "Una vez determinados los tipos de unidades de registro y de contexto, se pasa a la fase llamada codificación. Tales datos, son el conjunto de unidades de registro detectadas en los textos que deberán ser adscritas a sus respectivas unidades de contexto. Una vez codificadas las unidades pueden ser contabilizadas y relacionadas".

En el análisis por categorización Bardin (2002), plantea la importancia del contenido por medio de categorías conceptuales, desde las denominadas familias 
conceptuales, ya que, lo importante es determinar los conceptos de las palabras, conceptos que van más allá de la palabra en sí, sino que, apunta al significado particular del concepto.

Podemos reconocer el análisis de contenido por medio de categorización, como "la técnica que permite investigar el contenido de las "comunicaciones" mediante la clasificación en "categorías" de los elementos o contenidos manifiestos de dicha comunicación o mensaje” (Aigneren, 1999, pág. 4).

Según se ha expresado en términos teórico conceptuales, antes de considerar y elaborar las categorías respectivas, debemos saber identificar las unidades de análisis de un análisis de contenido. Entre ellas identificamos tres centrales, la unidad de muestro, registro y contexto.

- Unidad de muestreo: "son las diversas partes de la realidad sometida a observación que el investigador considera como separadas e independientes entre sí".

- Unidad de registro: "es la mínima porción del contenido que el investigador aísla y separa por aparecer allí uno de los símbolos, palabras claves, slogan, o temas que el investigador considera significativas”.

- Unidad de contexto: "es el párrafo o frase en el que está situada la unidad de registro que define el significado preciso de la misma” (Aigneren, 1999, págs. 25, 26,27).

De esta forma podemos establecer la necesidad de distinguir entre las respectivas unidades registro y contexto, para luego categorizar realizando una reducción interpretativa, inferencial y conceptual de dichas unidades de análisis.

Para Aigneren (1999, pág. 32), “Las categorías son los casilleros entre los que se van a distribuir las unidades de registro para su clasificación y recuento. El establecimiento del sistema de categorías a utilizar en el análisis es indudablemente el elemento más importante de la infraestructura del Análisis de Contenido".

Según López A. citado por Aigneren (1999, pág. 33), la forma de obtención de categorías debe velar por ciertos requisitos, de los cuales destaca:

1.- Las categorías deben reflejar los objetivos planteados en la investigación, con variables definidas e indicadores específicos.

2.- Las categorías deben ser exhaustivas, donde cada unidad de registro debe corresponder a una determinada categoría.

3.- No se debe colocar una unidad de registro en más de una categoría, es decir, deben ser excluyentes.

4.- Las categorías son independientes entre sí.

5.- Las categorías derivan de principios de clasificación, los cuales permiten separar conceptualmente los niveles de análisis. 


\subsection{Análisis de contenido temático}

El análisis de contenido tuvo una importancia creciente en su aplicabilidad, a partir de los conflictos de la segunda guerra mundial y los estudios referentes a su propaganda (Bardin, 2002). La intención del análisis de contenido, es llevar a cabo un estudio del contenido manifiesto y posteriormente latente aplicado a diferentes tópicos y temas. También podemos conocer este tipo de análisis como análisis temático (Cáceres, 2003).

Existirían diversas tipologías de los análisis de contenidos, los cuales dependerían de los objetivos de la investigación, así como metodologías, unidades de análisis, etc. De esta forma, Arbeláez \& Onrubia (2014) refiriéndose al trabajo de Colle en 1988 y Andréu (2000), clasifican los análisis de contenido en temático, semántico y de redes. En este sentido, y coherente con el presente estudio, podemos entender el análisis de contenido temático como el que:

"considera la presencia de términos o conceptos con independencia de las relaciones surgidas entre ellos. Las técnicas más utilizadas son las listas de frecuencias; la identificación y clasificación temática; y la búsqueda de palabras en contexto. Dentro de estas técnicas se seleccionan y, en ocasiones, se definen los términos o conceptos antes de iniciar para precisar las unidades de análisis” (pág. 20).

Piñuel (2002) establece que en estas unidades de categorías de análisis temáticas, la disección de análisis son los conceptos o referencias, los que son utilizados en las áreas de psicología cognitiva, psicosociología y sociología de la comunicación. A su vez, su procesamiento o medida puede ser de tipo cuantitativo estadístico y/o cualitativos lógicos, donde los objetos de estudio podrían ser trabajos relativos a la comunicación interpersonal y de masas.

El procedimiento de los análisis de contenido temático podrían plantearse de dos formas, una de ellas la deductiva que busca en el texto categorías previamente establecidas y que se construyen mediante referentes teóricos. Y por otro lado, la inductiva, que se caracteriza por una construcción de categorías emergentes desde el contenido, y que por tanto se podrían inferir (Arbeláez \& Onrubia, 2014).

Los autores plantean que la importancia de la inducción en términos cualitativos, surge porque este comienza desde las lecturas del documento, intentando identificar ejes centrales o categorías temáticas principales de un texto. De esta forma, desde los propios documentos emergen a posteriori, los que debieran poseer diferencias entre los documentos (Arbeláez \& Onrubia, 2014).

Finalmente, para Andréu (2000) el análisis de contenido categorial temático, es una técnica más de este tipo de análisis, en la cual se utilizan los análisis de presencia y ausencia de términos o conceptos con independencia entre sí. 


\section{Metodología}

Según criterios propuestos por Piñuel (2002), Arbeláez \& Onrubia (2014) indican que el presente estudio se llevó a cabo con una clasificación de análisis de contenido según su objetivo descriptivo, el cual pretende identificar y por tanto catalogar el contenido de los artículos, a través del uso de definición de categorías. Según su diseño de estudio, el análisis adecuado es de tipo longitudinal, ya que, apunta hacia el análisis de un corpus "en diferentes momentos de su trayectoria, ya sea aplicando medidas repetidas o sirviéndose de muestras independientes. La idea es analizar el corpus siempre bajo los mismos parámetros para ver efectivamente la evolución de un corpus textual, lo que permitirá a su vez utilizarlo como indicador de cambio social” (2014, pág. 21). Según su parámetro de medición es cualitativo, en los cuales se analiza la presencia o ausencia de categorías, y según su unidad de registro y análisis es una unidad temática de conceptos y referencias, lo que permitirá en definitiva dilucidar la orientación temática de una plataforma de difusión científica.

Tal como refieren Arbeláez \& Onrubia (2014), se realizó un estudio de los artículos publicados en revista Universum, identificando temáticas emergentes técnicamente a posteriori de los trabajos publicados en 30 años de edición. Esto con el objeto que luego de detectar la emergencia temática, dichas categorías conceptuales se agrupen para poder llegar a establecer ciertas tendencias y aglomeraciones que nos permitan identificar significaciones y así interpretarlas en un período determinado. De esta forma, identificamos tendencias y sus respectivas variaciones dentro de un marco de tiempo. Por tanto, y tal como mencionamos en la discusión bibliográfica, para el caso del presente trabajo se procedió a una categorización de tipo inductiva, ya que, apelamos a una constitución de categorías temáticas a posteriori a medida que se procedía a la lectura de los artículos de la revista.

\subsection{Diseño de la investigación}

Según Valles (2000) y Marradi, Archenti \& Piovani (2007), se debe entender el diseño de investigación cualitativa, como el proceso por el cual se da forma a una investigación mediante fases específicas, lo que repercute también en la toma de decisiones teóricas y metodológicas que el investigador realiza previamente, durante y después del estudio, luego de haber recopilado la información necesaria durante el proceso de investigación práctica.

En este sentido, Valles (2000) parafraseando a Morse (1994) expone que dichos procesos son la fase de "reflexión" en la formulación del problema de investigación; fase de "planeamiento" donde aparece la importancia del contexto de la investigación, su estrategia metodológica y escritura del proyecto; fase de "entrada", donde aparecen los informantes y la aplicación de los instrumentos; fase de "recogida productiva y análisis preliminar"; fase de "salida de campo y análisis extenso"; y por último la fase de "escritura”. Por otro lado, se debe considerar que hay decisiones las cuales están fuertemente influenciadas por factores éticos e 
ideológicos, contenidas a partir de la praxis del investigador inmerso en su contexto histórico y sociocultural, para configurar su problema de investigación, tal como lo señala Janesick (1994) según el citado texto de Valles.

En relación al diseño de la "temática central del escrito", esta fue elaborada mediante un proceso de configuración categórica, el cual se llevó a cabo mediante el siguiente procedimiento metodológico:

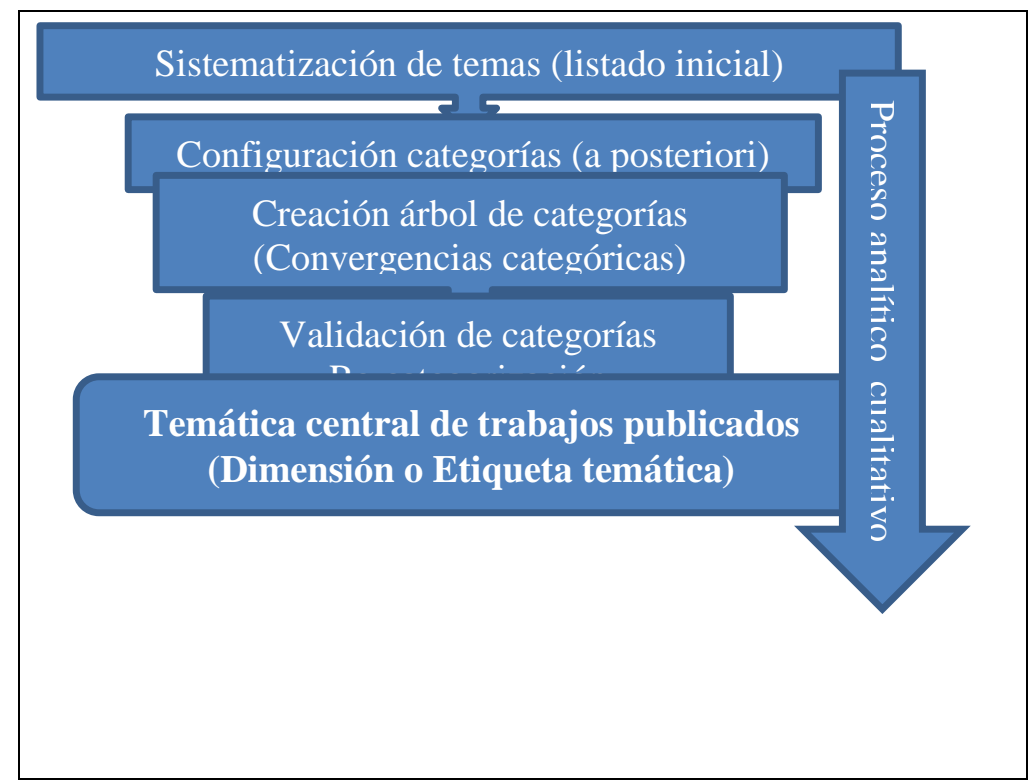

Figura $\mathrm{N}^{\mathrm{o}}$ 1: Diseño de análisis de contenido cualitativo.

Fuente: Elaboración propia.

\subsection{El tratamiento de la validez}

A partir de un proceso interpretativo de generación del saber, la validez del conocimiento se sustenta en la rigurosidad de la praxis investigativa. Se asume una posición donde la subjetividad e intersubjetividad del proceso investigativo da cuenta del trabajo, organización y decisiones que toma el investigador para la generación de los resultados (Cisterna, 2005).

El conocimiento del investigador respecto a fenómeno de estudio y por tanto, referente al análisis de contenido, hace que las decisiones que toma el analista tengan su valides en la medida de ser quién está directamente relacionado con la formación de categorías temáticas, según sus procedimientos de reducción de categorías (Arbeláez \& Onrubia, 2014).

En relación a lo anterior, para Cáceres (2003) la capacidad de agrupar ideas o componentes de forma integrada y coherente, es tarea fundamental del analista, ya que, corresponde a una decisión de carácter subjetivo e interpretativo, en la cual el investigador en razón de la profundidad de inmersión en el tema investigado, es capaz de dar sustento y validez a esta agrupación. 


\subsection{Muestra documental}

En términos metodológicos, la unidad de análisis se objetualiza como “documento", que según Moliner en 1984 citado en Valles (2000), se debe entender el mencionado "documento" según tres acepciones centrales:

1.- como un testimonio escrito el cual permite reconstruir historias pasadas.

2.- como un escrito el cual permite justificar o acreditar algo, por ejemplo un título, certificado, escritura notarial, contrato, etc.

3.- como un tipo de instrucción o enseñanza de alguna materia en particular.

En nuestro caso, se entendió como "documento" los aportes publicados en la revista Universum, en la cual se expresa una trayectoria de contenidos temáticos en cada una de las publicaciones de la revista en sus 30 años a la fecha.

Para definir el documento, nos quedaremos con quien a juicio de Valles (2000), es una de las mejores descripciones del término. Para ello, el autor cita a Mac Donald y Tipton (1993: 188), quienes plantean que los documentos son:

"cosas que podemos leer y que se refieren a algún aspecto del mundo social. Claramente esto incluye aquellas cosas hechas con la intención de registrar el mundo social -los informes oficiales, por ejemplo- pero también los registros privados y personales como cartas, diarios y fotografías [...] y que sin embargo, nos dicen algo sobre los valores intereses y propósitos de aquellos que la encargaron o produjeron” (pág. 120).

En definitiva se consideró la totalidad de publicaciones de la revista, las que contemplan finalmente 46 publicaciones anuales y bianuales entre los años 1986 y 2017. En total son 747 trabajos que poseen alguna autoría, sean estos ensayos; artículos científicos; reseñas bibliográficas; conferencia/clase magistral/discurso; entrevista; semblanza; o colaboración especial, las que terminaron por ser analizadas para el respectivo análisis de contenido y reducción de categorías que nos permitieron llegar a los resultados (Díaz, 2017).

En términos operativos de procesamiento de la información, y con el objeto de facilitar la reducción de categorías, se utilizó el software para análisis de datos “Statistical Package for the Social Sciences” (SPSS) en su versión 20.

\subsection{Procedimiento de análisis para la muestra documental}

Con el objeto de cumplir empíricamente con esta propuesta teórica - metodológica, el artículo propone un procedimiento que permite identificar una centralidad de contenidos que una vez aplicada la reducción de categorías conceptuales, nos entrega temáticas centrales de esta trayectoria de producción académica, en una revista regional adscrita a una de las instituciones de educación superior más importantes de Chile. 
Tabla $\mathrm{N}^{\circ}$ 1: Proceso de análisis de contenido temático.

\begin{tabular}{|c|c|}
\hline Proceso & Descripción del procedimiento \\
\hline $\begin{array}{l}1^{\circ} \text { Sistematización de } \\
\text { temas } \\
\text { (listado inicial) }\end{array}$ & $\begin{array}{l}\text { Una vez seleccionada la unidad de análisis de muestreo en extenso -en } \\
\text { nuestro caso revista Universum-, se procedió a generar un listado con los } \\
\text { títulos, resumen y palabras claves de los trabajos publicados. }\end{array}$ \\
\hline $\begin{array}{c}2^{\circ} \text { Configuración de } \\
\text { categorías } \\
\text { (a posteriori) }\end{array}$ & $\begin{array}{l}\text { En este procedimiento, se establecen las unidades de registro más } \\
\text { elementales de cada uno de los textos, separando como recomienda Bardin } \\
(2002) \text {, los elementos más significativos, que puedan dar cuenta en } \\
\text { términos generales, del texto completo, es decir, hablamos de un parte del } \\
\text { contenido que pueda representar el sustrato temático del trabajo publicado. } \\
\text { Este procedimiento puede llevarse mediante una labor inferencial a partir } \\
\text { de una lectura complementaria de título, palabras claves, resumen, artículo } \\
\text { completo y bibliografía presentada. } \\
\text { En este procedimiento se realiza un tipo de análisis inductivo, desde donde } \\
\text { emergen categorías a posteriori (Arbeláez \& Onrubia, 2014) que van a ser } \\
\text { identificadas y sistematizadas en un nuevo listado. } \\
\text { Para facilitar el trabajo empírico, se utilizó el programa SPSS con el objeto } \\
\text { de lograr identificar a nivel de frecuencias las temáticas recurrentes de las } \\
\text { publicaciones de la revista. Sin embargo, y para los fines específicos de } \\
\text { este procedimiento, se puede utilizar cualquier programa estadístico que } \\
\text { nos permita identificar niveles de frecuencias. }\end{array}$ \\
\hline $\begin{array}{c}3^{\circ} \text { Creación árbol de } \\
\text { categorías }\end{array}$ & $\begin{array}{l}\text { En este paso se procede a aglutinar las categorías, en familias conceptuales } \\
\text { creadas y cuyo nombre es adoptado según las temáticas emergentes de los } \\
\text { artículos. Estas temáticas sustentan significativamente conceptos centrales } \\
\text { extraídos de cada uno de los trabajos publicados. } \\
\text { Posteriormente, podremos denominar como “dimensiones" o “etiquetas" a } \\
\text { estas familias conceptuales que contienen este conglomerado de categorías } \\
\text { emergentes (a posteriori) del procedimiento descrito con anterioridad. }\end{array}$ \\
\hline $\begin{array}{c}4^{\circ} \text { Validación de } \\
\text { categorías } \\
\text { (Re-categorización) }\end{array}$ & $\begin{array}{l}\text { Una vez creado el árbol de categorías, el cual surge del ordenamiento del } \\
\text { listado preliminar de los trabajos publicados, se procede a la confección de } \\
\text { una re-categorización de estas temáticas, con el objeto de realizar una } \\
\text { reducción de categorías atingentes y coherentes con los temas que van } \\
\text { emergiendo y aglomerándose en este listado preliminar. } \\
\text { Este acto re-interpretativo de categorías temáticas emergentes, otorgan un } \\
\text { proceso de validez a la construcción de categorías preliminares, según los } \\
\text { planteamientos mencionados por Arbeláez \& Onrubia (2014) y Cáceres } \\
\text { (2003). }\end{array}$ \\
\hline $\begin{array}{l}5^{\circ} \text { Temática central de } \\
\text { publicación }\end{array}$ & $\begin{array}{l}\text { Finalmente y producto de los procedimientos anteriores, emerge un } \\
\text { conjunto de etiquetas o dimensiones temáticas centrales y periféricas, cuya } \\
\text { centralidad a nivel de frecuencia permite identificar la orientación temática } \\
\text { e intelectual de diversas producciones científicas, en nuestro caso las } \\
\text { publicaciones de Universum. }\end{array}$ \\
\hline
\end{tabular}

Fuente: Elaboración propia.

En consecuencia y en la tabla anterior de lectura descendente, se expresa por etapas cada uno de los procedimientos llevados a cabo para la reducción de dimensiones, con el objeto de comenzar desde la construcción del listado inicial, hasta finalizar con la creación de etiquetas o dimensiones posicionadas jerárquicamente, las que emanan de un proceso de categorización y recategorización cualitativa, gracias a una reducción de categorías mediante un procesamiento inferencial e interpretativo. 


\subsection{Características técnicas de profesionales que lleven a cabo la propuesta teórico-metodológica}

En razón de una propuesta teórica - metodológica, para llevar a cabo los procedimientos de categorización y re-categorización, se requieren de ciertas competencias, habilidades y/o capacidades que investigadores, equipos de trabajo o estudiantes, debieran cumplir mínimamente. Esto con el objeto de velar constantemente por la valides en cada uno de los procedimientos llevados a cabo. Según lo anterior quien suscribe, así como los investigadores a realizar dicha labor se recomienda que:

- Hayan realizado cursos de metodología de la investigación de enfoque cuantitativo y cualitativo. Esto con el objeto que los fundamentos y toma de decisión en cada uno de los procesos de categorización y re-categorización, sea sustentado en términos técnicos, teóricos y epistemológicos.

- Posean conocimientos técnicos en uso de software que permitan sistematizar a nivel de frecuencias los resultados que se van generando a posteriori (emergente o inductiva) o a priori (deductiva).

- Tengan conocimientos técnicos y teóricos en análisis de contenido cualitativo y cuantitativo.

- Hayan desarrollado capacidad para realizar inferencias de textos en términos cualitativos e interpretativos.

- Posean comprensión lectora que permita identificar elementos medulares o centrales de un documento.

- Idealmente puedan tener experiencia en el desarrollo de investigación cualitativa con análisis de contenido.

- Tengan capacidad de generar de manera estructurada y sistematizada, reportes con los resultados.

- Puedan profundizar y apropiarse respecto a las características y tipo de documento que se analizará, esto significa realizar revisiones preliminares, sistemáticas y conocer el documento antes de sistematizarlo preliminarmente.

\section{Resultados}

Creación de categorías en análisis de contenido cualitativo.

Según los pasos señalados con anterioridad (ver tabla $\mathrm{N}^{\circ} 1$ ), se generó como resultado una columna denominada "temática preliminar" a través de un procedimiento a posteriori (inductiva) que podemos identificar en la segunda columna de la siguiente tabla $\mathrm{N}^{\circ} 2$. De esta forma, los 747 títulos publicados en la revista, son reducidos y por tanto categorizados en 37 re-categorizaciones que darán vida a las futuras etiquetas o dimensiones.

Una vez contempladas esas 37 re-categorizaciones, se procede inferencialmente a una posterior reducción que da cuerpo a un nuevo y último proceso de re- 
categorización, resultando en total 11 temas centrales re-categorizados (ver tabla $\mathrm{N}^{\mathrm{o}}$ 3) que pasan a ser jerarquizados para cumplir con nuestro objetivo.

Tabla № 2. Proceso de categorización temática y posterior re-categorización.

\begin{tabular}{|c|c|c|c|c|}
\hline $\mathbf{N}^{\mathbf{0}}$ & Temática preliminar & $\begin{array}{l}\text { Frecuencia } \\
\text { documentos }\end{array}$ & $\begin{array}{c}\% \\
\text { equivalente }\end{array}$ & Re-categorización \\
\hline 1 & $\begin{array}{l}\text { Política/Teo. } \\
\text { Sociopolítica / Relaciones } \\
\text { internacionales }\end{array}$ & 88 & 11,8 & $\begin{array}{l}\text { Teorías social, política } \\
\text { y cultural }\end{array}$ \\
\hline 2 & Literatura & 74 & 9,9 & Humanismo y letras \\
\hline 3 & Economía histórica & 59 & 7,9 & $\begin{array}{l}\text { Desarrollo económico } \\
\text { y administración }\end{array}$ \\
\hline 4 & $\begin{array}{l}\text { Cultura / Teoría } \\
\text { sociocultural }\end{array}$ & 54 & 7,2 & $\begin{array}{l}\text { Teorías social, política } \\
\text { y cultural }\end{array}$ \\
\hline 5 & Historia e Historiografía & 52 & 7,0 & Humanismo y letras \\
\hline 6 & Epistemología / Filosofía & 51 & 6,8 & Humanismo y letras \\
\hline 7 & $\begin{array}{l}\text { Intelectuales / Ideas / } \\
\text { circulación de ideas }\end{array}$ & 40 & 5,4 & Estudio de las ideas \\
\hline 8 & $\begin{array}{l}\text { Pensamiento social, } \\
\text { económico, político }\end{array}$ & 39 & 5,2 & Estudio de las ideas \\
\hline 9 & $\begin{array}{l}\text { Expresiones de identidad } \\
\text { e imaginarios }\end{array}$ & 39 & 5,2 & $\begin{array}{l}\text { Teorías social, política } \\
\text { y cultural }\end{array}$ \\
\hline 10 & Educación & 33 & 4,4 & Humanismo y letras \\
\hline 11 & Homenaje & 31 & 4,1 & Homenaje \\
\hline 12 & $\begin{array}{l}\text { Expresiones del Arte / } \\
\text { Historia del arte }\end{array}$ & 26 & 3,5 & Humanismo y letras \\
\hline 13 & $\begin{array}{l}\text { Lenguaje, Argumentación } \\
\text { y Discursividad }\end{array}$ & 20 & 2,7 & Humanismo y letras \\
\hline 14 & Derecho y Cs. Jurídicas & 19 & 2,5 & Ciencias jurídicas \\
\hline 15 & $\begin{array}{l}\text { Comunicaciones, prensa y } \\
\text { Periodismo }\end{array}$ & 16 & 2,1 & $\begin{array}{l}\text { Ciencias de la } \\
\text { comunicación }\end{array}$ \\
\hline 16 & $\begin{array}{l}\text { Socio Economía y } \\
\text { desarrollo urbano - rural }\end{array}$ & 12 & 1,6 & $\begin{array}{l}\text { Desarrollo económico } \\
\text { y administración }\end{array}$ \\
\hline 17 & Patrimonio / Arquitectura & 11 & 1,5 & $\begin{array}{l}\text { Teorías social, política } \\
\text { y cultural }\end{array}$ \\
\hline 18 & $\begin{array}{l}\text { Espacio y Geografía } \\
\text { urbano- rural }\end{array}$ & 9 & 1,2 & $\begin{array}{l}\text { Desarrollo económico } \\
\text { y administración }\end{array}$ \\
\hline 19 & $\begin{array}{l}\text { Industria, trabajo y } \\
\text { empleo }\end{array}$ & 9 & 1,2 & $\begin{array}{l}\text { Desarrollo económico } \\
\text { y administración }\end{array}$ \\
\hline 20 & $\begin{array}{l}\text { Ciencia, Investigación, } \\
\text { Metodología y Técnicas }\end{array}$ & 7 & ,9 & $\begin{array}{l}\text { Teorías social, política } \\
\text { y cultural }\end{array}$ \\
\hline 21 & $\begin{array}{l}\text { Manifestaciones y } \\
\text { sistemas religiosos }\end{array}$ & 7 & ,9 & $\begin{array}{l}\text { Teorías social, política } \\
\text { y cultural }\end{array}$ \\
\hline 22 & $\begin{array}{l}\text { Lingüística / } \\
\text { Sociolingüística / } \\
\text { Filología }\end{array}$ & 6 & ,8 & Humanismo y letras \\
\hline
\end{tabular}




\begin{tabular}{|c|c|c|c|c|}
\hline 23 & $\begin{array}{l}\text { Salud, Bienestar y } \\
\text { Calidad de Vida }\end{array}$ & 6 & ,8 & $\begin{array}{l}\text { Teoría de la psique y } \\
\text { Psicológica aplicada }\end{array}$ \\
\hline 24 & Forestal & 5 & ,7 & $\begin{array}{ll}\text { Ciencias biológicas, } \\
\text { físicas y del medio } \\
\text { ambiente }\end{array}$ \\
\hline 25 & Botánica & 5 & ,7 & \begin{tabular}{ll} 
Ciencias & \multicolumn{2}{c}{ biológicas, } \\
físicas y del medio \\
ambiente
\end{tabular} \\
\hline 26 & Arqueología & 4 & ,5 & $\begin{array}{l}\text { Teorías social, política } \\
\text { y cultural }\end{array}$ \\
\hline 27 & $\begin{array}{l}\text { Ecología y medio } \\
\text { ambientalismo }\end{array}$ & 4 & ,5 & \begin{tabular}{ll} 
Ciencias & \multicolumn{2}{c}{ biológicas, } \\
físicas y del medio \\
ambiente
\end{tabular} \\
\hline 28 & Tecnología y navegación & 3 & ,4 & \begin{tabular}{ll} 
Ciencias & \multicolumn{2}{c}{ biológicas, } \\
físicas y del medio \\
ambiente
\end{tabular} \\
\hline 29 & $\begin{array}{l}\text { Administración, } \\
\text { ingeniería, gestión y D.O }\end{array}$ & 3 & ,4 & $\begin{array}{l}\text { Desarrollo económico } \\
\text { y administración }\end{array}$ \\
\hline 30 & Desarrollo hidrográfico & 3 & ,4 & $\begin{array}{ll}\text { Ciencias biológicas, } \\
\text { físicas y del medio } \\
\text { ambiente }\end{array}$ \\
\hline 31 & Medicina / Inmunología & 3 & ,4 & \begin{tabular}{ll} 
Ciencias & \multicolumn{2}{c}{ biológicas, } \\
físicas y del medio \\
ambiente
\end{tabular} \\
\hline 32 & Lecto - escritura & 2 & ,3 & Humanismo y letras \\
\hline 33 & Astronomía / Física & 2 & ,3 & $\begin{array}{l}\text { Ciencias biológicas, } \\
\text { físicas y del medio } \\
\text { ambiente }\end{array}$ \\
\hline 34 & $\begin{array}{l}\text { Era digital, tecnología, } \\
\text { cibernética, web }\end{array}$ & 2 & , 3 & $\begin{array}{l}\text { Mundo digital } \mathrm{y} \\
\text { tecnológico }\end{array}$ \\
\hline 35 & Psiquiatría & 1 & ,1 & $\begin{array}{l}\text { Teoría de la psique y } \\
\text { Psicológica aplicada }\end{array}$ \\
\hline 36 & $\begin{array}{l}\text { Archivos y gestión de } \\
\text { información }\end{array}$ & 1 & ,1 & Bibliotecología \\
\hline 37 & $\begin{array}{l}\text { Teorías de estructuras } \\
\text { mentales }\end{array}$ & 1 & ,1 & $\begin{array}{l}\text { Teoría de la psique y } \\
\text { Psicológica aplicada }\end{array}$ \\
\hline
\end{tabular}

Fuente: elaboración propia.

Con el objeto de reducir las categorías temáticas, estas pueden ser aglutinadas entre sí según pertinencia temática y teórica. Resulta así una reducción a 11 temáticas que revista Universum ha desarrollado en su trayectoria de tres décadas desde el año 1986 a 2017. Este ejercicio se ajusta a un proceso inferencial y de recategorización temática, que a partir de este momento, dicha re-categorización final podemos denominar como “etiquetas temáticas” o “dimensiones temáticas”, ya que, sería el resultado final del procedimiento dando sustento a la orientación temática de un trabajo cienciométrico en este caso particular, para una producción académica de frecuencia periódica. 
Tabla N ${ }^{\circ}$ 3. Orientación temática de la re-categorización final: Creación de dimensiones o etiquetas temáticas.

\begin{tabular}{|l|l|l|}
\hline $\mathbf{N}^{\mathbf{0}}$ & \multicolumn{1}{|c|}{$\begin{array}{c}\text { Dimensión temática o Etiqueta temática } \\
\text { (Re-categorización) }\end{array}$} & $\mathbf{\%}$ \\
\hline 1 & Humanismo y letras & 35,4 \\
\hline 2 & Teorías social, política y cultural & 28 \\
\hline 3 & Desarrollo económico y administración & 12,3 \\
\hline 4 & Estudio de las ideas & 10,6 \\
\hline 5 & Homenaje & 4,1 \\
\hline 6 & Ciencias biológicas, físicas y del medio ambiente & 3,4 \\
\hline 7 & Ciencias jurídicas & 2,5 \\
\hline 8 & Ciencias de la comunicación & 2,1 \\
\hline 9 & Teoría de la psique y Psicológica aplicada & 1 \\
\hline 10 & Mundo digital y tecnológico & 0,3 \\
\hline 11 & Bibliotecología & 0,1 \\
\hline
\end{tabular}

Fuente: elaboración propia.

En este sentido y tal como se expresa en la tabla $\mathrm{N}^{\mathrm{o}} 3$, el total de estas 11 dimensiones temáticas -y gracias a un proceso de jerarquización de dichas etiquetas-, puede darnos a conocer una orientación temática o intelectual para esta producción científica de referencia. De esta forma, la jerarquía de los resultados da cuenta de cuatro dimensiones centrales que se han desarrollado en la revista, cuya lectura descendente dice que en primera instancia revista Universum posee una dimensión predominante en humanismo y letras (35,4\%); luego teoría social, política y cultural (28\%); desarrollo económico y administración (12,3\%); y estudio de las ideas $(10,6 \%)$. Con posterioridad a esta centralidad temática de cuatro etiquetas, las siete dimensiones restantes se disgregan a nivel periférico, complementando así el total de 11 etiquetas emergentes re-categorizadas del total de la revista.

\section{Discusión}

El propósito del análisis de contenido se encuentra en "la inferencia de conocimiento relativos a las condiciones de producción (o eventualmente a recepción), con ayuda de indicadores (cuantitativos o no)” (Bardin, 2002, pág. 29). La idea para el autor, es la inferencia que pueda deducir de manera lógica los conocimientos respecto al emisor del mensaje o bien su contexto. Por ende, se requiere en primera instancia “describir” (enumeración de características) y por último, la "interpretación” (inferir los procedimientos del texto), lo que sin lugar a dudas resulta fundamental y relevante para este tipo de trabajos. De esta forma, conceptos como inferencia y categorización se transforman en relevantes a la hora de un procedimiento inductivo a posteriori.

Tal como refiere Andréu (2000) al centrarse en procedimientos reductivos de las categorías, se lograron crear criterios de definición, las cuales se van 
deduciendo sistemáticamente hasta lograr una categoría central, lo que podemos traducir en una labor inductiva del análisis de contenido. Lo que en definitiva permite ser configurado a través de mecanismos metodológicos sistemáticos y estrictamente procedimentales. La revisión de categorías y constante evaluación de las reducciones categóricas, se transforman en un procesos de valides cualitativa para la emergencia de las temáticas inductivas.

Según lo anterior y a partir de trabajos como los de Arbeláez \& Onrubia (2014), Olvera (2016), Ramos (2005), Andrade (2015), Luna Ledesma (2015) y Navarro \& Moyano (2017) podemos dar cuenta de la importancia que puede revestir el análisis de revistas dedicadas al pensamiento de las ciencias sociales y humanas, así como un análisis de las orientaciones temáticas, epistemológicas y metodológicas en un área de estudios en particular.

Se buscó contribuir a dilucidar la construcción de un saber, en una revista periódica con sus respectivas tendencias u orientaciones intelectuales, la que se caracteriza por una génesis multidisciplinaria e informativa en una casa de estudios en particular de Chile, además esta publicación se contextualizarla en un marco institucional de floreciente proceso de modernización.

Según lo anterior, esta investigación contempló un análisis de contenido cualitativo que coherente al objetivo del estudio, aporta a la supuesta discusión entre posturas cuantitativas o cualitativas del análisis de contenido en investigación social; y por otro lado, transformarse en un aporte teórico - metodológico en esta técnica de análisis temática.

Con el objeto de seguir otorgando legitimidad a la siguiente propuesta y a mayor abundamiento, Moyano \& Ramos (2000) realizan un ejercicio metodológico cualitativo de categorización, dentro de un estudio cuantitativo de variables categóricas respecto a la forma en cómo se está produciendo conocimiento psicológico en Chile, desde una perspectiva cienciométrica. Los resultados presentados del estudio son de carácter cuantitativo, a nivel porcentual del total de artículos y de los títulos de revistas en el área psicológica, así como tipos de trabajo ya sean revisiones teóricas, trabajos aplicados, trabajos mixtos, de divulgación, reflexión teórica, expansión teórica y por especialidad psicológica, entendiéndose estos dentro de psicología básica y experimental, clínica, educacional, social - ambiental o de trabajo y organizacional.

Por su lado, Devés (2008) lleva a cabo un procedimiento en el cual a través de la creación de seis modelos, explica la circulación de ideas hacia regiones periféricas. La construcción de estos modelos resultantes fueron en definitiva: Instituciones académicas del primer mundo; Encuentros de científicos económicos-sociales; Redes sobre cuestiones del desarrollo; Estadías de latinoamericanos en Asia y África; Conferencias y congresos internacionales y Publicaciones.

Estos ejemplos dan cuenta que el análisis de contenido temático de textos, producción científica o encuentros académicos, pueden expresar una trayectoria intelectual, científica, u orientación normativa de un área de estudio, por tanto, resulta ser un procedimiento que se realiza habitualmente en ciencias sociales, 
dándole mayor realce y valides a la propuesta procedimental, metodológica y técnica para estos fines.

En términos empírico analíticos, el estudio nos entregó posterior al análisis de contenido categorizado y temático, un cúmulo de dimensiones por los cuales se expresa la trayectoria de la revista Universum, este tipo de dimensiones o áreas del conocimiento logradas tras el ejercicio interpretativo temático de la reducción de categorías, nos da luces de la disposición multidisciplinar de la revista, que si bien es cierto, circunda incluso entre dimensiones físico-naturales, digitales, bibliotecología, arqueología y otras áreas, existe un predominio de las áreas de las humanidades ciencias sociales, dando coherencia al subtítulo de la revista que se adjudica a partir del año 2004, cuando la editorial de la revista anuncia su indexación a la plataforma Scielo (Pinedo, 2004).

Se logró realizar una bajada empírica a los planteamientos metodológicos anteriores, analizando la trayectoria y aportes publicados en una revista académica de 30 años de historia, logrando identificar las dimensiones centrales por las cuales se sustenta la orientación intelectual de los aportes de la revista.

En definitiva, el artículo se configuró en tres partes esenciales, la primera de ellas sustentando una discusión bibliográfica que de luces de las formas como ingresar desde una perspectiva interpretativa, hacia un enfoque cualitativo al análisis de contenido y sin dejar de mencionar los requerimientos epistemológicos previos al acercamiento de la investigación cualitativa y la necesaria mención al supuesto antagonismo metodológico. De esta forma, la trayectoria epistemológica sustenta un método de investigación cualitativa hasta llegar a las técnicas de análisis de contenido, con sus respectivos mecanismos de abordaje como la categorización temática mencionada y trabajado por autores como Bardin (2002).

En una segunda configuración, el trabajo elabora un mecanismo o procedimiento técnico y metodológico por el cual, desde el análisis de contenido temático y por categorización, se puede generar un procedimiento válido que complemente los trabajos elaborados desde la cienciometría. En este sentido y mediante este tipo de análisis de contenido cualitativo, podemos elaborar o desentrañar dimensiones temáticas del desarrollo o producción académica en diferentes formas de expresión, llámese artículos de revistas, publicaciones de actas científicas de congresos, mesas de trabajo de estos encuentros académicos, trayectoria intelectual de autores referentes, etc.

\section{Conclusiones}

Podemos concluir a raíz de la pregunta inicial, la que pretende dilucidar cuál es la orientación temática y por tanto intelectual de una revista regional afiliada a la Universidad de Talca (región del Maule - Chile), que son cuatro las dimensiones principales por las cuales circunda el desarrollo intelectual y de difusión de la revista, las que identificamos a nivel central con las etiquetas: a) Dimensión Humanista y Letras; b) Dimensión Teórico Social, Político y Cultural; c) Dimensión Desarrollo Económico y Administración; y d) Dimensión Estudio de las 
Ideas. A continuación podemos identificar en un segundo nivel el resto de etiquetas, las que dentro del desarrollo de la publicación han bordeado a nivel periférico la orientación de la revista, dentro de las cuales se encuentra: e) Homenaje; f) Ciencias Biológicas, Físicas y del Medio Ambiente; g) Ciencias Jurídicas; h) Ciencias de la Comunicación; i) Teoría de la Psique y Psicológica Aplicada; j) Mundo Digital y Tecnológico; y por último k) Bibliotecología. Es decir, cuatro etiquetas centrales que en definitiva dilucidan una orientación temática intelectual central y siete etiquetas de orden periférico.

De esta forma damos cuenta de una trayectoria de tres décadas, que fiel a su origen multidisciplinar si bien, abrió las puertas a otras áreas del conocimiento, durante el transcurso de los años definió su orientación intelectual hasta su declaración en el año 2004 cuando ingresa a indexación Scielo, definiéndose como una revista científica de humanidades y ciencias sociales. Esto nos invita a reflexionar respecto a la importancia de las indexaciones en las publicaciones periódicas, las que dan sustento a criterios de calidad y orientación temática, que logramos identificar una vez realizados los análisis de contenido respectivos de estas publicaciones. Por otro lado, podemos dar cuenta de la coherencia interna y editorial de la revista, por tanto este tipo de trabajo puede servir para evaluaciones de gestión de la producción científica, cara a acreditaciones, control de gestión, evaluaciones de desempeño institucional o editorial, etc.

Si bien el valor de esta revista que en primera instancia tiene un carácter regional y nacional, su desempeño constante determinó una gestión editorial que logró internacionalizarse ingresando a la plataforma Scopus, y con ello, asume el desafío que constituye expandirse a otros idiomas para su publicación, según refiere en entrevista el mismo director Dr. Javier Pinedo. De esta forma, pasa a ser una revista de carácter internacional y de proyección científica de alto impacto.

Finalmente, el procedimiento aplicado desde un análisis de contenido temático y cualitativo, puede ayudar a cimentar una orientación del desarrollo intelectual temático de un área de estudio. Esta técnica de análisis cualitativo llevado a cabo bajo estos procedimientos, ayuda a complementar aportes de un trabajo cienciometrico, que pueden desentrañar desde un composición interpretativa y de forma válida, las orientaciones temáticas de producción científica, abriendo las posibilidades a análisis de otras formas de producción, como literatura, publicaciones de encuentros académicos, congresos, seminarios, entre otras.

\section{Agradecimientos}

Agradecimientos especiales al Dr. Emilio Moyano y Dr. Iván Pérez por sus valiosos comentarios al presente artículo; al Dr. Eduardo Devés por su orientación a la investigación doctoral; al Dr. Javier Pinedo, por su apoyo y entrevista para el estudio; a la Dra. Leyla Torres y Biblioteca central de la Universidad de Talca (campus Lircay), por su gestión y disposición a facilitar las revistas publicadas. 


\section{Referencias Bibliográficas}

Aigneren, M. (1999). Análisis De Contenido. Una Introducción. Revista La Sociología en sus Escenarios.

Andrade, A. (2015). La Revista Mexicana de Ciencias Políticas y Sociales 60 años de apoyo a la producción científica. Revista mexicana de ciencias políticas y sociales, 61(226), 457 - 472.

Andréu, J. (2000). Las técnicas de Análisis de Contenido: Una revisión actualizada. Fundación Centro Estudios Andaluces - Universidad de Granada, 10(2), 1 - 34.

Araya, S. (2002). Las representaciones sociales: Ejes teóricos para su discusión. San José, Costa Rica: Facultad Latinoamericana de Ciencias Sociales (FLACSO) .

Arbeláez, M., \& Onrubia, J. (2014). Análisis bibliométrico y de contenido. Dos metodologías complementarias para el análisis de la revista colombiana Educación y Cultura. Revista de Investigaciones UCM, 14(23), 14 - 31.

Bardin, L. (2002). El análisis de contenido. Madrid: Ediciones Akal.

Bernal, C. (2006). Metodología de la investigación: Para administración, economía, humanidades y ciencias sociales. México: Ediciones Pearson.

Cáceres, P. (2003). Análisis cualitativo de contenido: Una alternativa metodológica alcanzable. Psico perspectivas, II, 53 - 82.

Cisterna, F. (2005). Categorización y triangulación como procesos de validación del conocimiento en investigación cualitativa. Theoria, 14(1), 61 - 71.

De Sierra, G., Garretón, M. A., Murmis, M., \& Trindade, H. (2007). Las ciencias sociales en América Latina en una mirada comparativa. En H. Trindade (Ed.), Las ciencias sociales en América Latina en perspectiva comparada (págs. 17-52). México: Editorial Siglo XXI.

Delgado, J., \& Gutiérrez, J. (1998). Métodos y técnicas cualitativas de investigación en ciencias sociales. Madrid: Editorial Síntesis.

Devés, E. (2008). La circulación de las ideas económico-sociales de Latinoamérica y el Caribe, en Asia y África ¿Cómo llegaron y cómo se diseminaron? Universum, 2, 87111.

Devés, E. (2016). Una agenda para las ciencias económico-sociales y el pensamiento nuestramericano: Calidad, innovación, pertinencia, fidelidad, incidencia internacional y otras cosas más. Jornadas UDELAR, (pág. 21). Montevideo.

Díaz, C. (2014). Un acercamiento a la comprensión del método de investigación en ciencias sociales y jurídicas: el recorrido desde la naturaleza humana, los paradigmas y su método, en un contexto disciplinar dominado por la racionalidad teleológica. Revista Pedagogía universitaria y Didáctica del Derecho, 1(2), 80-104.

Díaz, C. (2017). Informe de caracterización de los 30 años de Revista Universum. Talca, Chile: Instituto de Estudios Humanísticos - Universidad de Talca.

Flores, J., García, E., \& Rodríguez, G. (1996). Metodología de la investigación cualitativa. Málaga: Editorial Aljibe.

Giddens, A. (1999). En defensa de la sociología. Madrid: Alianza.

Guba, E., \& Lincoln, Y. (2002). Paradigmas en competencia en la investigación cualitativa. En C. Denman, \& J. Haro, Por los rincones. Antología de métodos cualitativos en la investigación social (págs. 113-145). Hermosillo Sonora: Editorial El Colegio de Sonora.

Habermas, J. (1989). Teoría de la acción comunicativa II. Crítica de la razón funcionalista. Buenos Aires: Taurus. 
Hernández, R., Fernández, C., \& Baptista, P. (2010). Metodología de la investigación. México D.F: Editorial Mc Graw Hill.

Krippendorff, K. (2002). Metodología de análisis de contenido. Teoría y práctica. Barcelona: Paidós.

Luna, M. (2015). Sobre el futuro de las revistas de ciencias sociales: Elementos para la reflexión. Revista mexicana de sociología, 77(Supl. 1), 11 - 31.

Marradi, A., Archenti, N., \& Piovani, J. (2007). Metodología de las ciencias sociales. Buenos Aires: Editorial Emecé.

Moyano, E., \& Ramos, N. (2000). Contexto y evaluación de la investigación psicológica en el Chile de fin de siglo. Psykhe, 9(1), 63 - 75.

Navarro, J., \& Moyano, E. (2017). Metodología, temas y disciplinas en la investigación actual sobre migración internacional. Sociedade e Cultura, 20(2).

Noguera, J. (2010). El mito de la sociología como «ciencia multiparadigmática». Revista de Filosofía Moral y Política, 31 - 53.

Olvera, M. (2016). La etapa inicial de la Revista Mexicana de Ciencias Políticas y Sociales a 60 años de distancia Prácticas conmemorativas, legados olvidos y nuevos pasados/presentes. Revista Mexicana de Ciencias Políticas y Sociales, 61(226), 427 456.

Pinedo, J. (2004). Editorial Revista Universum. Universum, 7 - 8.

Piñuel, J. L. (2002). Epistemología, metodología y técnicas del análisis de contenido. Estudios de Sociolingüística, 3(1), 1-42.

Ramos, C. (2005). Cómo investigan los sociólogos chilenos en los albores del siglo XXI: Paradigmas y herramientas del oficio. Persona y Sociedad, XIX(3), 85-119.

Ritzer, G. (2001). Teoría sociológica clásica. Madrid: Mc Graw Hill.

Ritzer, G. (2002). Teoría sociológica moderna. Madrid: Mc Graw Hill.

Ruíz, J. (2012). Metodología de la investigación cualitativa. Bilbao: Universidad de Deusto.

Stake, R. (1999). Investigación con estudio de caso. Madrid: Ediciones Morata.

Tejedor, C. (1993). Historia de la filosofía en su marco cultural. España: SM.

Tylor, S., \& Bogdan, R. (1987). Introducción a los métodos cualitativos de investigación. Barcelona: Editorial Paidos.

Valles, M. (2000). Técnicas cualitativas de investigación social. Madrid: Editorial Síntesis. Weber, M. (2005). Economía y Sociedad. México: Editorial Fondo de Cultura Económica . 